\title{
Frakturen und Luxationen der unteren HWS
}

\author{
Michael Oberst, Bernd Wittner, Ulrich Holz
}

\section{Zusammenfassung}

Verletzungen der unteren HWS sind oft instabil. Neurologische Begleitverletzungen treten relativ häufig auf. Bei vielen dieser Verletzungen besteht eine absolute Operationsindikation, bei etlichen konkuriert die operative mit der konservativen Behandlung. Die konservative Behandlung erfolgt durch Ruhigstellung im Halofixateur für 6-8 Wochen. Je nach Verletzungsmuster kann die operative Versorgung von ventral, dorsal oder kombiniert erfolgen.

\section{Einleitung}

Die Abschnitte der Wirbelsäule vom Bewegungssegment $\mathrm{C} 2 / \mathrm{C} 3$ bis zum Segment C7/Th1, also vom Oberrand der Bandscheibe $\mathrm{C} 2 / \mathrm{C} 3$ bis zum Unterrand der Bandscheibe C7/Th1 zeigen biomechanisch ein ähnliches Verhalten und ein pathologisch-anatomisch ähnliches Bild. Dieser Teil der Wirbelsäule unterscheidet sich durch seine einheitliche Anatomie, seine Funktion, die beobachteten Verletzungsmuster und den Richtlinien zu ihrer Behandlung von den beiden ersten Halswirbeln einerseits und von den nachfolgenden thorakalen Wirbeln andererseits, so dass dieser Abschnitt der Wirbelsäule in der Klinik zur „Unteren HWS“ zusammengefasst wird. Verletzungen in diesem Wirbelsäulenabschnitt sind relativ häufig und oft mit einem neurologischen Ausfall kombiniert.

OP-JOURNAL 2001; 17: 142-149

(c) Georg Thieme Verlag Stuttgart · New York

\section{Anatomie}

Im Bereich der unteren HWS hat es sich aufgrund der speziellen Anatomie als sinnvoll erwiesen, das 3-Säulen-Konzept nach Denis et al. zugunsten eines 2-Säulen-Konzeptes zu modifizieren. Abb.1 zeigt die schematische Darstellung der Komponenten der unteren HWS in eine vordere und eine hintere Säule, getrennt durch das hintere Längsband. Der Spinalkanal ist im Bereich der unteren HWS deutlich schmäler als im Bereich der oberen beiden Wirbel (Abb.2), was mit verantwortlich für die relative Häufigkeit von Schäden am Rückenmark bei Verletzungen dieses Wirbelabschnittes ist.

\section{Epidemiologie und Verletzungenmechanismus}

Fast $4 / 5$ aller Verletzungen der Halswirbelsäule betreffen die untere HWS, am häufigsten den 5. Halswirbelkörper und das Bewegungssegment C5/C6. Im Vergleich zur thorakolumbalen Wirbelsäule sind bei Verletzungen der unteren HWS die dorsalen ligamentären Strukturen durch Distraktion viel häufiger verletzt, so dass in einem höheren Prozentsatz instabile Verletzungen vorliegen. Andererseits erklärt der hohe Anteil an Luxationsfrakturen das vermehrte Auftreten von neurologischen Schäden. Neurologische Schäden bei Verletzungen an der Wirbel-

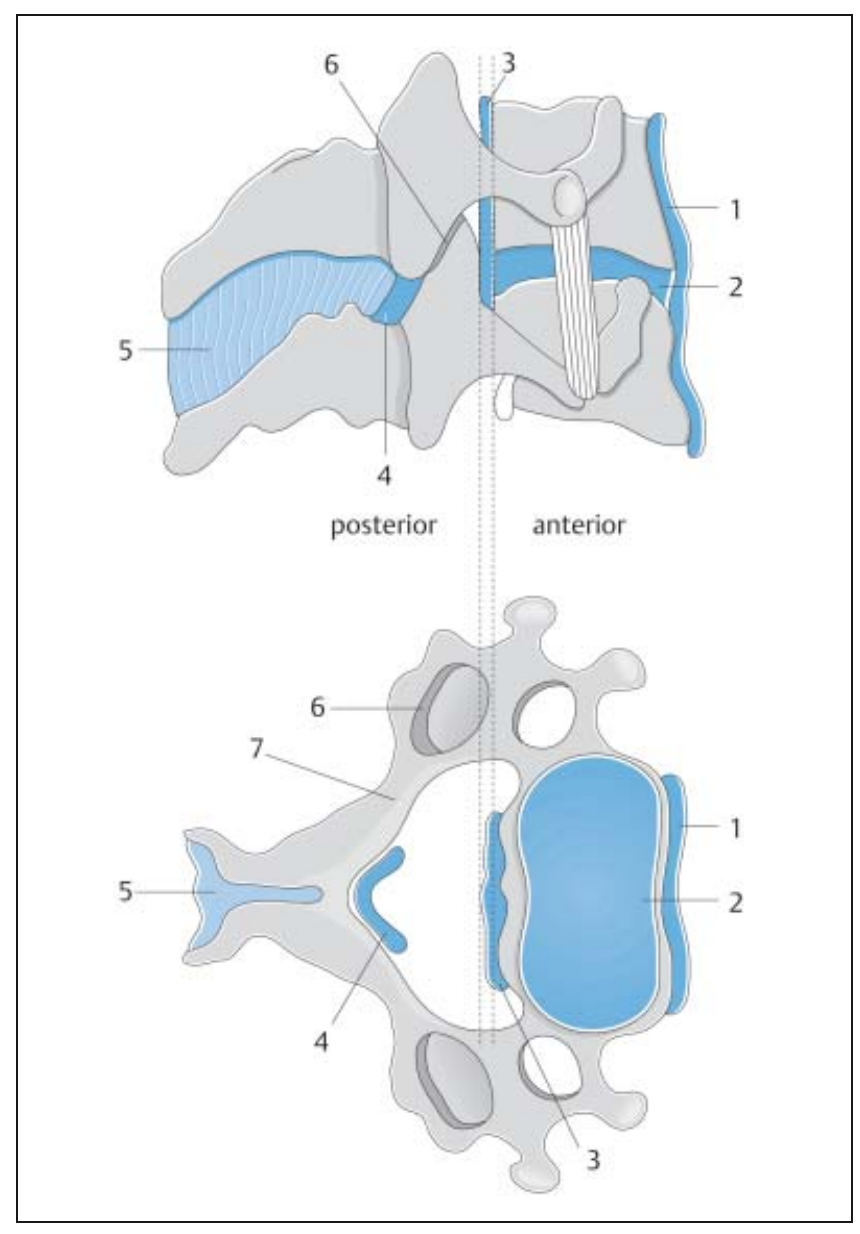

Abb. 1 Der Aufbau der Wirbel von C3C7 ist sehr ähnlich. Dieser Abschnitt wird daher im klinischen Gebrauch zur „Unteren HWS“ zusammengefasst. (1 vorderes Längsband, 2 Bandscheibe, 3 hinteres Längsband, 4 Lig. flavum, 5 Lig. interspinosum, 6 Gelenkfortsatz, 7 Lamina). Aus „Wirbelsäulenverletzungen" (E.-J. Müller, G. Muhr) Thieme Stuttgart, 1997, S. 66. 


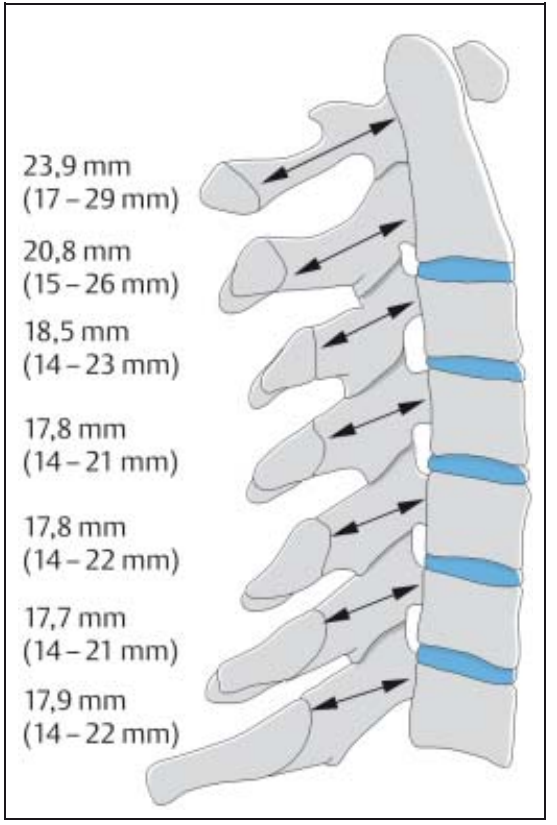

Abb. 2 Der Wirbelkanal ist im Bereich der unteren HWS deutlich schmäler als in Höhe der Wirbel C1 und C2. Aus Blauth/Tscherne: Unfallchirurgie - Wirbelsäule Springer Berlin, 1998.

säule finden sich an der unteren HWS in $40 \%$, während nur $15-20 \%$ der Verletzungen der thorakalen und lumbalen Wirbelsäule mit neurologischen Ausfällen einhergehen (Benson; Keenen 1990). 55\% aller Verletzungen der Wirbelsäule mit neurologischen Schäden betreffen die HWS. Komplexe Verletzungen mit Beteiligung des Wirbelkörpers, der kleinen Gelenke, der Bandstrukturen und/oder der Bandscheiben sind an der HWS deutlich häufiger als an der übrigen Wirbelsäule.

Verletzungen der HWS sind häufiger mit Zerreißungen der dorsalen Bänder kombiniert, haben häufiger neurologische Begleitschäden und stellen im Mittel instabilere Verletzungen dar als die traumatischen Schäden an der BWS und HWS.

Die Verletzung entsteht zumeist durch indirekte Gewalteinwirkung auf den Kopf oder den Körper. Ein typischer Unfallhergang ist ein Kopfsprung ins seichte Wasser. Durch den Aufprall des Körpers wird dieser abrupt gestoppt, während die kinetische Energie des Körpers die Halswirbelsäule einer massiven Krafteinwirkung aussetzt. Anhand von Leichenstudien konnte gezeigt werden, dass bei solchen Ereignissen Kräfte bis über $10000 \mathrm{~N}$ (>1 Tonne) von den Strukturen der Halswirbelsäule aufgefangen werden müssen (Crowell et al. 1989).

\section{Diagnostik}

In aller Regel ergibt sich aus der Schilderung des Unfallherganges durch den Verletzten bzw. beim bewusstseinsgetrübten Patienten durch Augenzeugen oder das Rettungspersonal der Hinweis, ob eine HWS-Verletzung vorliegen könnte. Bei jedem bewusstlosen Verletzten besteht primär der Verdacht auf eine HWS-Verletzung, so dass die HWS dieser Patienten solange mit einer steifen Orthese geschützt wird, bis die weitere Diagnostik eine Verletzung sicher ausgeschlossen hat. Besonders häufig sind HWS-Verletzungen mit folgenden Befunden assoziiert, die nicht selten vom Erscheinungsbild des Verletzten im Vordergrund stehen:

Schädel-Hirn-Trauma, Gesichtsschädelverletzungen, Opfer eines Verkehrsunfalls und Bewusstlosigkeit.

Bei jedem bewusstlosen Verletzten besteht primär der Verdacht auf eine Verletzung der HWS, so dass bei jedem dieser Patienten die HWS durch eine steife Orthese solange geschützt wird, bis die weitere Diagnostik eine Verletzung sicher ausgeschlossen hat.

Besonders erfragt werden müssen jegliche Hinweise auf temporäre oder persistierende neurologische Symptome. Die klinische Untersuchung beginnt mit der Palpation der HWS, ohne dass dabei der Kopf des Verletzten bewegt wird. Beim bewusstseinsklaren Verletzten macht eine schmerzfreie Palpation der Dornfortsätze eine bedeutende HWS-Verletzung sehr unwahrscheinlich. Während der Untersuchung achtet man außer auf Schmerzäußerungen besonders auch auf Stufen und Lücken in der Dornfortsatzreihe sowie auf lokale Hämatombildungen. Nach der lokalen Untersuchung wird der Verletzte eingehend neurologisch untersucht. Beim bewusstseinsklaren Patienten werden die Motorik, die epikritische und gnostische Sensibilität in jeder Höhe und die Reflexe beidseits geprüft und schriftlich dokumentiert. Beim bewusstseinsgetrübten Verletzten werden die Qualitäten untersucht, die sinnvoll untersuchbar sind. Auch bei Halswirbelsäulenverletzungen gehört zum neurologischen Status eine rektale Untersuchung mit Beurteilung des Sphinktertonus, der perianalen Sensibilität und des Bulbocavernosusreflexes. Besonders bei einer motorischen und sensiblen Querschnittssymptomatik hat die rektale Untersuchung große Bedeutung um bei vermeintlicher Tetraplegie eine „Zentrale Rückenmarksläsion“, eine Sonderform der imkomplette Parese zu erkennen, bei der die „Sakrale Innervation" erhalten bleibt (Sacral spars.) (Tab.1) Ergibt die weitere Diagnostik eine signifikante Verletzung der Halswirbelsäule, so wird die neurologische Untersuchung im selben Ausmaß wiederholt, um Veränderungen zu erfassen.

Eine eingehende neurologische Diagnostik einschließlich rektaler Untersuchung ist Bestandteil der Diagnostik jedes Verletzten mit einer signifikanten Halswirbelsäulenläsion.

Die apparative Untersuchung beginnt mit einem Röntgenbild der Halswirbelsäule seitlich, das die Wirbelsäule vom Hinterhaupt bis zur Deckplatte des ersten Brustwirbels darstellen muss. Hierzu ist es in aller Regel notwendig, dass die Aufnahme unter leichtem Zug an den Armen ausgeführt wird. Gelingt es nicht damit die ganze HWS darzustellen, müssen Schrägaufnahmen des zervikothorakalen Übergangs angefertigt werden (Abb.3). Gelingt es auch damit nicht eine ausreichende Darstellung des siebten Halswirbels und des Bewegungssegments C7/Th1 zu erreichen, ist eine CT-Untersuchung dieser Region unabdingbar. Dann wird eine a.p. Aufnahme der HWS angefertigt. Bei der Beurteilung der HWS-Aufnahmen achtet man auf:

- Einen Verlust der physiologischen Lordose der HWS

Tab. 1 Sensible und motorische Ausfallserscheinungen entsprechend der Höhe der Schädigung der HWS.

\begin{tabular}{lll} 
verletzte Höhe der HWS & sensibler Ausfall & motorischer Ausfall \\
\hline C4 & Schulterhöhe & Zwerchfell \\
C5 & Oberarmaußenseite & Ellenbogenbeuger \\
C6 & Daumen & Handgelenksstrecker \\
C7 & Mittelfinger & Ellenbogenstrecker \\
C8 & Kleinfinger & Fingerbeuger \\
Th1 & Innenseite des Ellenbogens & Fingerspreitzer
\end{tabular}




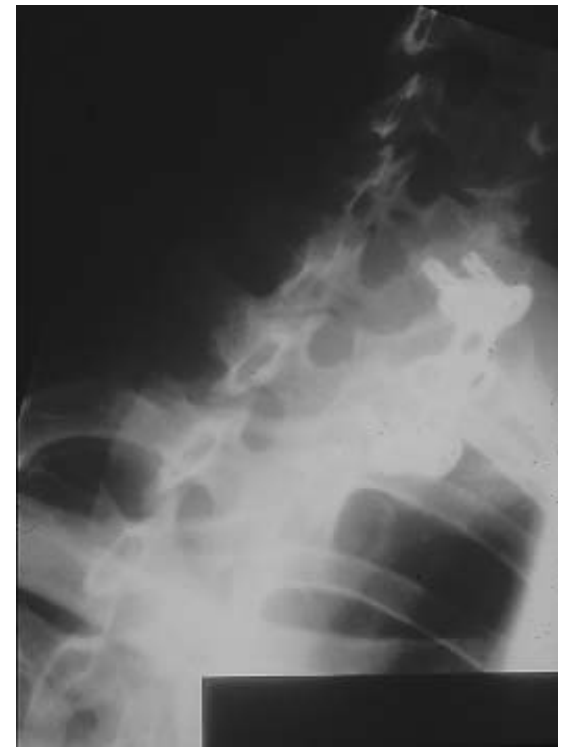

Abb. 3 Gelingt es nicht, den Wirbel C7 und den Übergang auf Th1 im seitlichen Bild darzustellen, sind Schrägaufnahmen zur Darstellung des Alignments der Wirbel hilfreich (postoperative Aufnahme nach Spondylodese C7 Th).

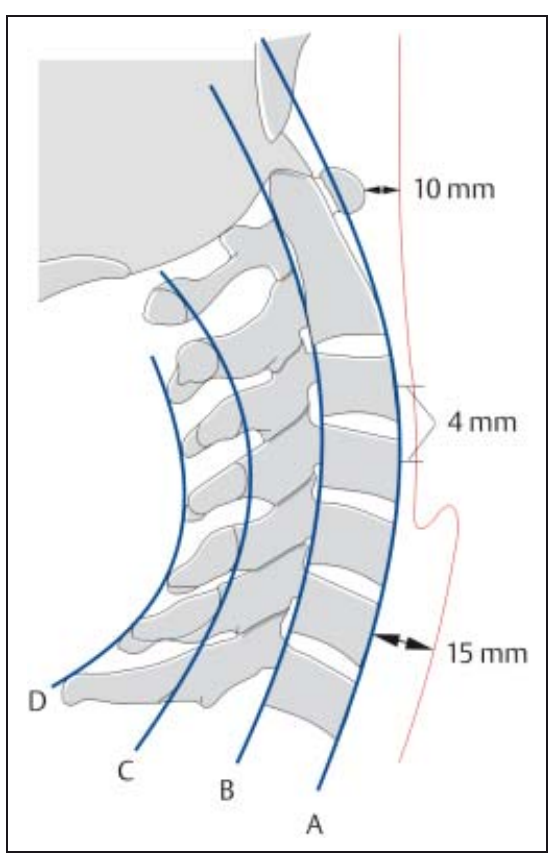

Abb. 4 Die vier Alignmentlinien der HWS müssen harmonisch verlaufen: Jede Stufe oder Unterbrechung bedarf der weiteren Abklärung. Aus Blauth/Tscherne a. a. O.

- Kyphotische Knickbildungen

- Frakturen der knöchernen Strukturen (Wirbelkörper, Massa lateralis, Lamimae, Gelenk- und Dornfortsätze)

- Verlust der vier cervikalen Alignmentlinien im seitlichen Bild (Abb.4)

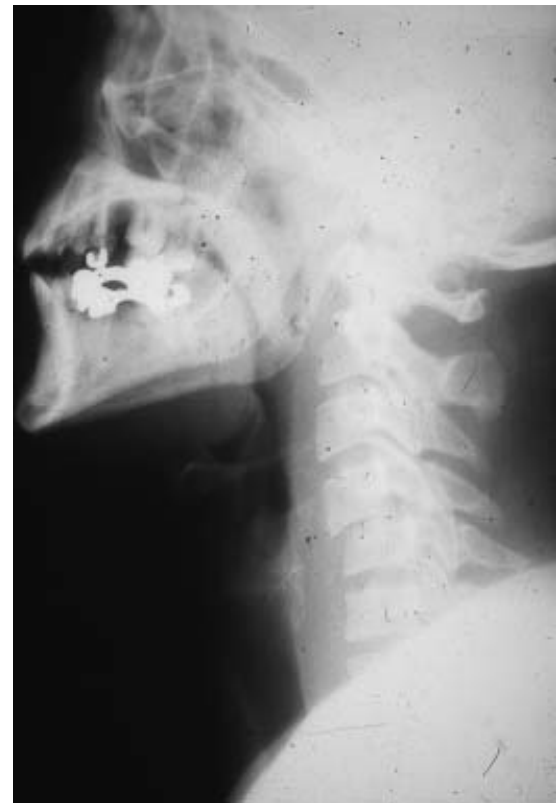

Abb. 5 Luxation im Bewegungssegment C4/ C5: Bei Verletzungen der HWS kommt es zur Hämatombildung hinter dem Pharynx. Was sich gut im Röntgenbild als Verbreiterung des prävertebralen Weichteilschattens darstellt.

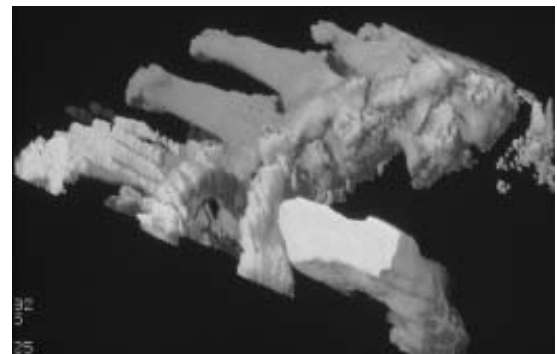

Abb. 6 Luxationsfraktur C5/6: 3D-Rekonstruktionen aus den CT-Daten verbessern das Verständnis.

- Verlust des Alignments der Dornfortsatzreihe im a.p. Bild

- Vermehrter Abstand der Dornfortsätze im a.p. oder seitlichen Bild

- Verbreiterter prävertebraler Weichteilschatten (max $10 \mathrm{~mm}$ vor dem 3. HWK und $\max 4 \mathrm{~mm}$ vor dem 5.HWS (Abb.5)

Jede nachgewiesene knöcherne Verletzung sollte heute mit einer axialen CTSchichtung und sagittaler ggf. koronarer Rekonstruktion bei Verwendung eines Spiral-CT weiter abgeklärt werden. 3DRekonstruktionen erleichtern die Vorstellung der Verletzung und die operative Planung, erhöhen jedoch nicht den Aussagewert der axialen Schichten (Abb.6).
Funktionsaufnahmen der frischverletzten HWS können diskoligamentäre Verletzungen aufzeigen, sie dürfen aber bei frischer Verletzung nur von einem erfahrenen Traumatologen unter Bildwandlerkontrolle ausgeführt werden. Beim wachen Patienten können sie aufgrund der Muskelverspannung falsch negativ ausfallen!

Funktionsaufnahmen der frisch verletzten HWS dürfen nur von einem erfahrenen Traumatologen unter Bildwandlerkontrolle durchgeführt werden. Beim wachen Patienten können sie falsch negativ ausfallen!

Die MRT-Untersuchung stellt zwar Läsionen der dorsalen Weichteilstrukturen dar, eine Aussage über die biomechanische Bedeutung dieser Verletzung ist jedoch nicht immer möglich. Die Hauptindikation für eine MRT-Untersuchung der verletzten HWS ist der Ausschluss eines OP-pflichtigen intraspinalen Hämatoms. Auch traumatische Bandscheibenvorfälle lassen sich am besten mit dem MRT nachweisen. Ergeben sich bei einer transienten oder permanenten neurologischen Symptomatik mit den bisherigen Untersuchungen kein Hinweis auf die Ursache, so ist immer eine Kernspinuntersuchung indiziert.

\section{Klassifikation der Verletzungen}

Die AO-Einteilung der Halswirbelsäulenverletzung geht auf Magerl et al. (1994) zurück. Zum Verständnis der AO-Klassifikation werden an der HWS eine ventrale (Wirbelkörper, Bandscheiben und ventrales Längsband) und eine dorsale Säule (Massae laterales, Laminae, Gelenkfortund Dornfortsätze sowie dorsaler Bandkomplex) unterschieden. Die Grenze zwischen den beiden Säulen bildet das hintere Längsband (Abb.1). Bei der Klassifikation werden unterschieden (Abb.7):

- Typ A: Kompressionsverletzung des Wirbelkörpers mit intakten dorsalen Band- und Knochenstrukturen (Kompressionsverletzung der ventralen Säule)

- Typ B: Distraktionsverletzung der dorsalen oder ventralen Säule. Die Distraktionsverletzung der dorsalen Säule ist oft mit einer Kompressionsverletzung der ventralen Säule kombiniert (Zweisäulenverletzung mit Distraktion)

- Typ C: Rotationsverletzung. (Zweisäulenverletzung mit Rotation). Da es bei den Rotationsverletzungen immer zu einer mehr oder weniger ausgeprägten 

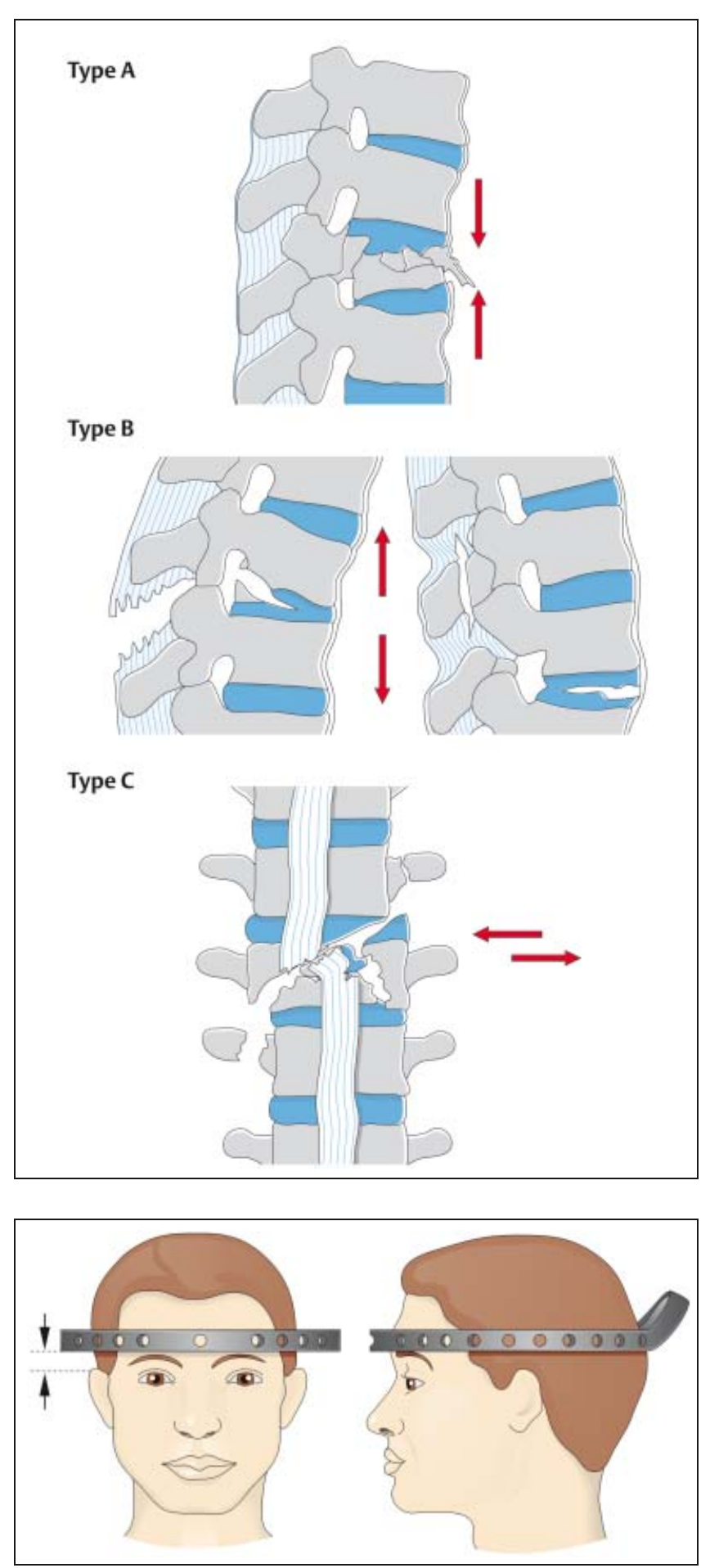

Abb. 7 Die AOKlassifikation unterscheidet drei Verletzungstypen: Typ A: Kompressionsverletzung; Typ B: Distraktionsverletzung; Typ C: Rotationverletzung. Aus O’Dowd, in Rüedi/ Murphy: AO-Principles of Fracture Management, Thieme Stuttgart, 2000.

Abb. 8 Bei der Montage eines Halofixateurs werden die ersten beiden Pins oberhalb der Augenbrauen und zwei direkt gegenüber am Hinterhaupt eingebracht. Aus O’Dowd a. a. O.
Translationsbewegung der Wirbelsäulenabschnitte gegeneinander kommt, sind dabei neurologische Schäden sehr häufig.
Innerhalb der Typen werden je nach Ausmaß der Verletzung entsprechend dem AO-Schema jeweils 3 Gruppen und innerhalb der Gruppen 3 Untergruppen von Verletzungen unterschieden. Diese Feinunterscheidung hat jedoch weniger Bedeutung für die Therapie und Prognose der Verletzung als die Einteilung nach den Typen A-C.
Die AO-Einteilung der HWS-Verletzungen unterscheidet drei Typen:

- Typ A: Kompressionsverletzung der ventralen Säule

- Typ B: Zweisäulenverletzung mit Distraktion

- Typ C: Zweisäulenverletzung mit Rotation

\section{Therapie}

\section{Primärbehandlung}

Initial wird bei jeder HWS-Verletzung die Halswirbelsäule mit einer steifen Orthese geschützt bis die Verletzung genau diagnostiziert ist. Findet sich im seitlichen Röntgenbild eine dislozierte Fraktur und zeigt der Verletzte neurologische Symptome bzw. ist der neurologische Status beim bewusstlosen Verletzten nicht sicher bekannt, so sollte ggf. parallel zur weiteren Basisdiagnostik die Reposition der Verletzung erfolgen. Diese wird durch die Montage eines Haloringes erheblich erleichtert (Abb.8). Die Reposition sollte möglichst direkt unter Bildwandlerkontrolle oder im Halofixateur mit ansteigendem Zug erfolgen. Fast jede Verletzung der unteren HWS lässt sich nach der Reposition mit einer steifen Krawatte und Fixation des Kopfes mit Sandsäcken ausreichend stabilisieren, selten ist hierzu ein Dauerzug am Haloring mit 3-4 kp nötig. Zur Stabilisierung bis zur definitiven Versorgung einer nicht dislozierten Fraktur ist eine steife Orthese immer ausreichend. Der Nutzen einer Behandlung der frischen Rückenmarkverletzung mit Methylprednisolon (Bolus von $30 \mathrm{mg} / \mathrm{kg} \mathrm{KG}$ gefolgt von $5,4 \mathrm{mg} / \mathrm{kg}$ $\mathrm{KG} \times \mathrm{h}$ für die nächsten 24 Stunden) ist zwar noch nicht endgültig bewiesen, trotzdem wenden wir das Schema bei jedem Patienten mit frischer Querschnittsymptomatik nach HWS-Verletzung an. Zeigt die obligate CT-Untersuchung nach der Reposition noch eine verbliebene mechanische Kompression des Rückenmarkes durch eingesprengte Knochenfragmente sollte die operative Entlastung des Rückenmarks und gleichzeitig Stabilisierung der Wirbelsäule so schnell wie möglich statt finden.

\section{Indikation zur Operation}

Über die Indikation zur operativen Versorgung von HWS-Verletzungen herrscht in der Literatur noch keine Einigkeit. 
Unbestrittene Indikationen (absolute Indikationen) sind:

- Komplette Tetraplegie. Funktionelle bedeutende Verbesserungen der Beinfunktion nach initial kompletter Tetraplegie sind zwar extrem selten, eine Erholung der Armfunktion um ein oder zwei Höhen wird jedoch häufig beschrieben, wobei diese Verbesserung nach operativer Reposition und Stabilisierung häufiger beobachtet wird als bei konservativer Behandlung. Der Zugewinn bereits eines Levels im Bereich der unteren HWS stellt für den Verletzten einen großen funktionellen Gewinn dar. Weiterhin wird die nachfolgende Pflege eines Patienten mit Tetraplegie bei stabil versorgter HWS-Verletzung deutlich erleichtert.

- Wurzelausfall bei im CT oder MRT nachgewiesener verbliebener mechanischer Kompression nach der Reposition. Da Nervenwurzeln im Gegensatz zum Rückenmark ein gutes Erholungspotential besitzen und diese Erholung besser ist, je früher die Dekompression der Wurzel erfolgt, stellt dieser Befund für uns eine absolute Notfallindikation zur operativen Entlastung und Stabilisierung dar.

- Progredientes Querschnittsyndrom bei im CT oder MRT nachgewiesener verbliebener mechanischer Kompression des Myelons. Im Gegensatz zur kompletten Tetraplegie zeigt die inkomplette Schädigung eine bessere Tendenz zur Erholung des Rückenmarks. Auch dieser Befund stellt für uns eine Indikation zur Notfalloperation dar.

- Sekundäre Verschlechterung der neurologischen Symptomatik. Resultiert die Verschlechterung auf einer sekundären Dislokation, so muss die Reposition sofort erfolgen. In diesen Fällen, schließen wir die operative Stabilisierung sofort an die Reposition an. Lässt sich eine Ursache für die Verschlechterung im Röntgenbild nicht erkennen, so ist eine MRT-Untersuchung notwendig um eine Myelonkompression durch Weichteile oder ein intraspinales Hämatom zu erkennen. Jede nachgewiesene mechanische Kompression stellt eine Indikation zur sofortigen operativen Entlastung dar.

- Ausgeprägte Instabilitäten eines Wirbelsegmentes. Ausgeprägte Zerreißungen der dorsalen Bänder heilen unter konservativer Behandlung selten stabil aus, so dass das entsprechende Bewegungssegment versteift werden sollte um chronischen Schmerzzuständen

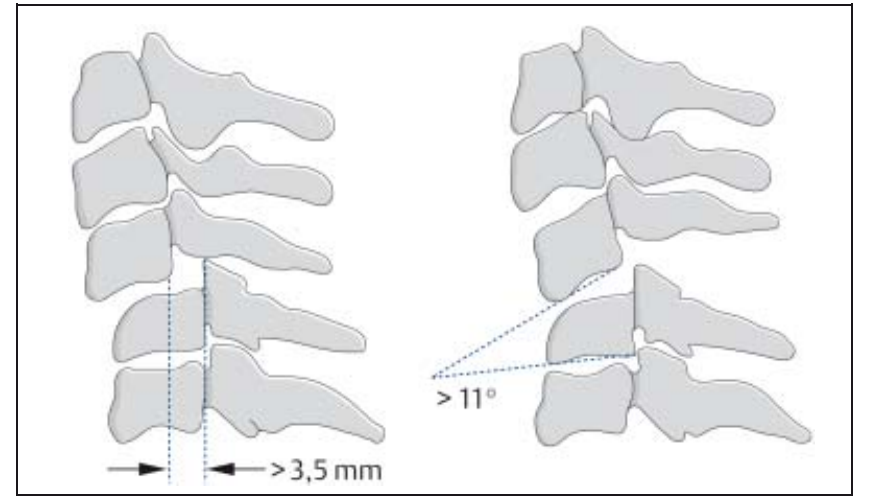

Abb. 9 Instabilitätskriterien nach White und Panjabi.

Aus E. J. Müller,

G. Muhr a. a. O.

vorzubeugen. Über diese Tatsache herrscht Einigkeit. Diskutiert wird jedoch welche Befunde für eine so massive Zerreißung der Bänder sprechen, dass daraus eine „ausgeprägte“ Instabilität resultiert. Eine Verschiebung zwischen zwei Wirbelkörpern um $4 \mathrm{~mm}$ und eine Knickbildung zwischen zwei Wirbelkörpern um mehr als $11^{\circ}$ bei intaktem Wirbelkörper (Abb.9, Kriterien nach White und Panjabi, 1990) werden im Allgemeinen als kleinster gemeinsamer Nenner anerkannt. Dabei ist jedoch zu beachten, dass einerseits die Kriterien nach White und Panjabi bei den häufigen Flexions-Kompressions-Verletzungen (Verletzungen der dorsalen Bandstrukturen mit gleichzeitiger Kompression des Wirbelkörpers), nicht angewendet werden können, da hierbei der kyphotische Knick nicht allein aus der Bandverletzung resultiert, sondern auch aus der Kompression der ventralen Säule. $\mathrm{Zu}$ anderen zeigt das Röntgenbild immer nur eine Momentaufnahme der Situation, auch nach ausgeprägten Verletzungen der Bandstrukturen kann es zur spontanen Reposition der Wirbel kommen, so dass die Instabilität im Standard-Röntgenbild nicht nachweisbar ist (Abb.10).

Unbestrittene Indikationen zur operativen Versorgung von Halswirbelsäulenverletzungen sind:

- Tetraplegie

- Wurzelausfall mit Kompression der Wurzel

- Progredientes Querschnittsyndrom bei Kompression des Myelons

- Sekundäre Verschlechterung der neurologischen Symptomatik

- Ausgeprägte Instabilität des Bewegungssegments
Weitere Operationsindikationen (relative Indikationen):

Knickbildungen der HWS im seitlichen Röntgenbild von mehr als $15^{\circ}$ bei radiologisch reinen Kompressionsfrakturen („radiologische“ Verletzungen des Typs A). Bei diesen Verletzungen ist in aller Regel die Bandscheibe zerstört und es muss in einem hohen Maß von zusätzlichen Verletzungen der dorsalen Bänder ausgegangen werden. Eine konservative Behandlung müsste für mindestens 6-8 Wochen im Halofixateur erfolgen (Abb.11). Die Reposition dieser Verletzungen ist konservativ nicht immer perfekt zu erreichen und sekundäre Korrekturverluste sind häufig. Wegen der radiologisch oft nicht diagnostizierbaren Bandverletzungen resultieren öfter chronische Instabilitäten.

- Verletzungen der dorsalen Bandstrukturen, die radiologisch einer geringeren Instabilität als den Kriterien nach White und Panjabl entsprechen. Auch hier wäre die konservative Behandlung im Halofixateur für 6-8 Wochen möglich. Die stabile Ausheilung wäre jedoch nicht gewährleistet (Abb.12).

- Knöcherne Einengung des Spinalkanals um mehr als $1 / 3$ nach der Reposition ohne neurologische Symptomatik. Eine Remodellierung des Spinalkanals nach verbliebener Einengung tritt zwar in der Regel ein, das Ausmaß dieser spontanen Korrektur ist jedoch nicht sicher vorhersehbar. Anhaltender chronischer Druck auf das Myelon führt zu degenerativen Veränderungen des Marks mit schleichender oft nicht rückbildungsfähiger Tetraparese. In diesen Fällen schlagen wir dem Verletzten die operative Dekompression des Rückenmarks und Stabilisierung vor auch wenn keine akute neurologische Symptomatik besteht. 

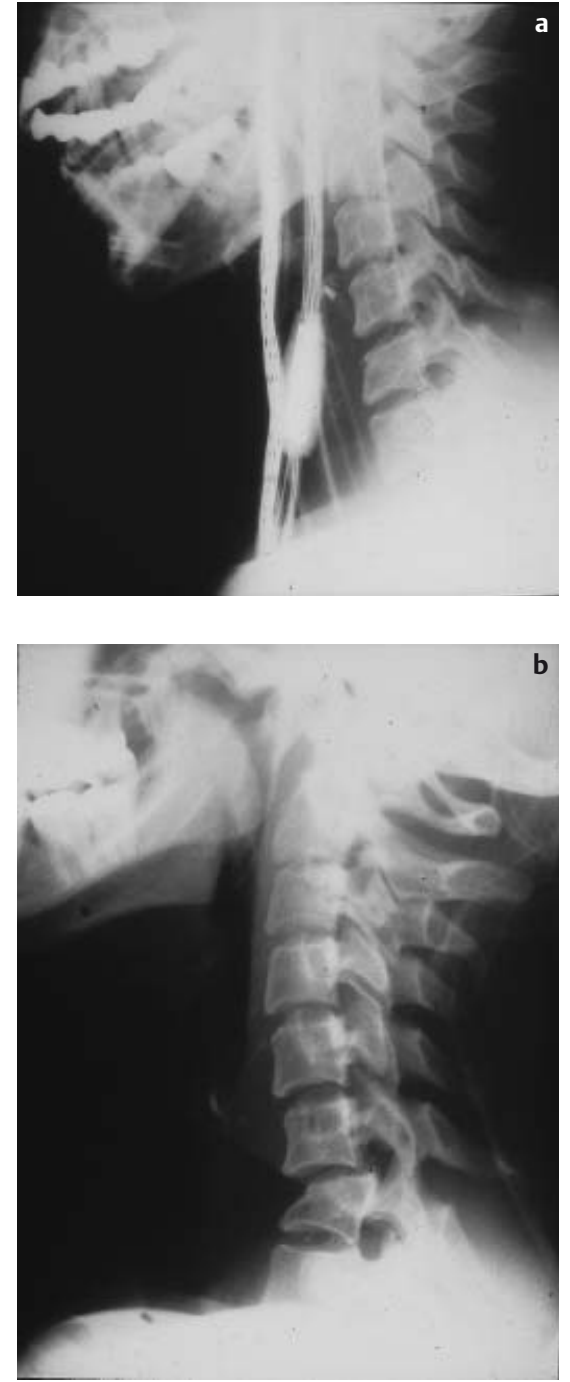

Abb.10 a Seitliches Röntgenbild der HWS vom Unfalltag: Hinweise auf eine Verletzung ergeben sich nicht, Funktionsaufnahmen wurden nicht angefertigt. b Nach Durchführung eines coronaren $\mathrm{CT}$, bei dem vom Verletzten der Kopf extrem rekliniert werden muss, fällt beim Patienten eine Tetraparese auf.

Empfehlenswerte Operationsindikationen sind:

Knickbildung der HWS im seitlichen Bild von mehr als $15^{\circ}$

- Verletzungen der dorsalen Bandstrukturen

- Knöcherne Einengung des Spinalkanals um mehr als $1 / 3$

Zusammenfassend empfehlen wir dem Patienten die operative Reposition und Stabilisierung bei allen Frakturen des Typs B und C sowie bei Frakturen des Typs A mit kyphotischem Knick über $15^{\circ}$, wobei der Verletzte selbstverständlich bei den relativen Indikationen ausführlich auch über die konservativen Be- handlungsalternativen im Halofixateur aufgeklärt werden muss.

Reine Kompressionsfrakturen mit Knickbildung unter $20^{\circ}$ und Fortsatzbrüche werden konservativ in der steifen Orthese behandelt. Die Dauer der Ruhigstellung schwankt zwischen wenigen Tagen und maximal 6 Wochen, je nach Beschwerden des Verletzten und spezieller Verletzung.

\section{Operative Technik}

Da Bandverletzungen an der Wirbelsäule meist nicht stabil ausheilen, besteht die operative Behandlung in der Regel in der Versteifung des betroffenen Bewegungssegmentes (Spondylodese), diese kann prinzipiell von ventral oder dorsal erfolgen.

Ventrale Spondylodese mit Ausräumung des Bandscheibenfaches und Interposition eines Beckenkammspan. Stabilisierung mit einer speziellen Platte, deren Design zur Erhöhung der Primärstabilität eine winkelstabile Verklemmung der Schrauben in der Platte ermöglicht (Abb.13).

Dorsale Spondylodese mit verschiedenen Implantaten (Hakenplatten, spezielle HWS-Platten, spezieller Fixateur intern für die HWS [Cervifix], reine Zuggurtungen der Dornfortsätze mit Drahtschlingen). Um eine Spondylodese zu erreichen, muss in diesen Fällen immer Spongiosa an die angefrischten knöchernen dorsalen Strukturen angelagert werden (Abb.14).

Kombiniert ventro-dorsale Spondylodese. Selten muss bei der Operation so-

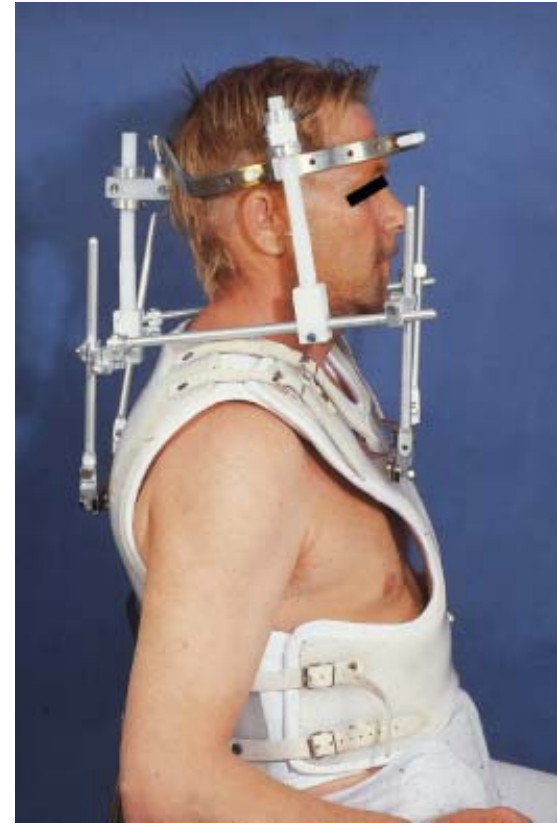

Abb.11 Bei vielen Verletzungen der HWS steht die operative Spondylodese gleichberechtigt neben der konservativen Behandlung mit Halofixateur.

wohl von dorsal wie ventral vorgegangen werden.

Die meisten HWS Verletzungen können sowohl von ventral wie von dorsal stabilisiert werden. Eine kombinierte ventrodorsale Stabilisierung ist nur selten nötig.

Die meisten HWS-Verletzungen können sowohl von ventral als auch von dorsal versorgt werden, wobei wir in diesen Fällen immer den ventralen Zugang favorisieren.

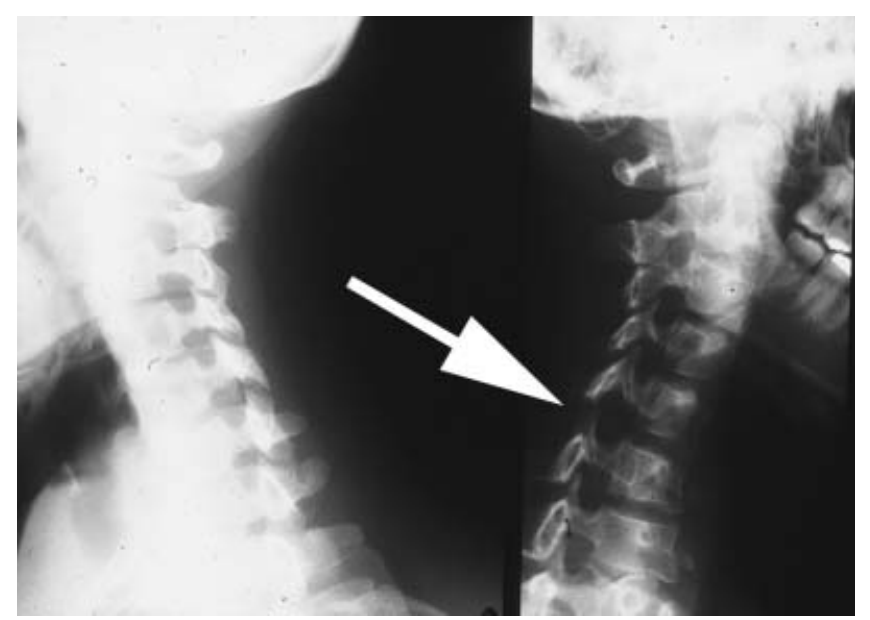

Abb.12 Einseitige Luxation der Wirbelgelenke: Eine konservative Behandlung dieser Verletzung für 6-8 Wochen im Halofixateur ist zwar möglich, eine stabile Ausheilung jedoch nicht immer erreichbar. Wir empfehlen dem Verletzten deshalb eine operative Stabilisierung dieser Verletzung. 


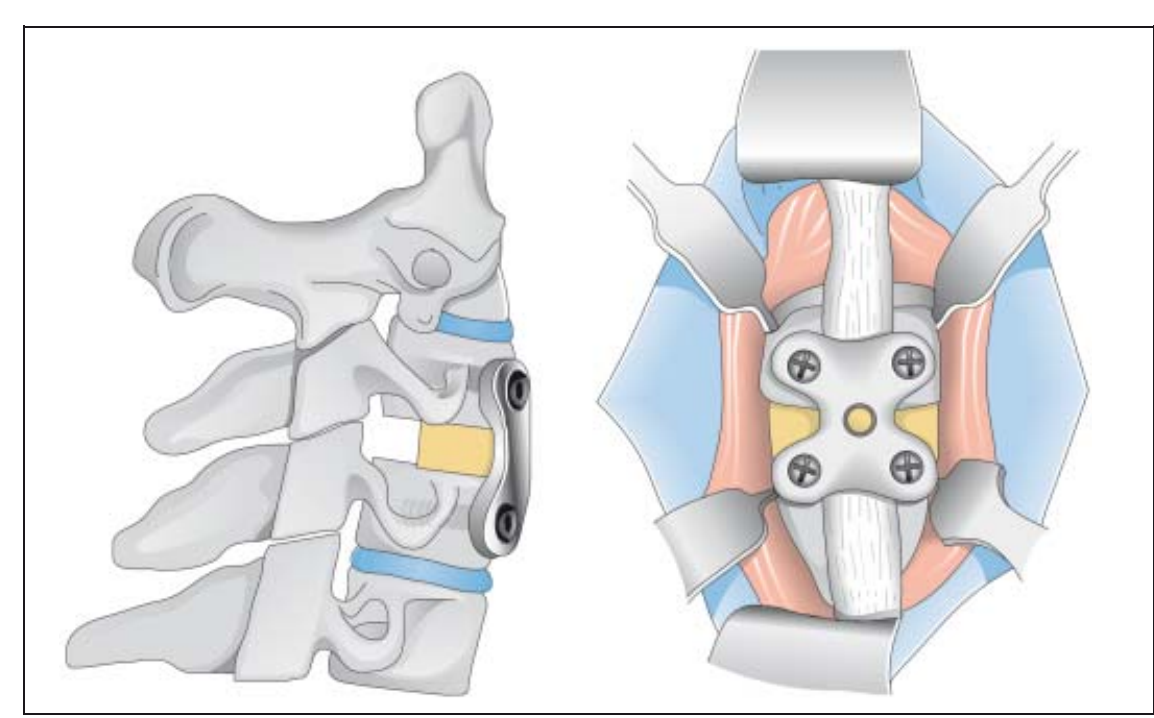

Abb.13 Ventrale Spondylodese mit winkelstabiler H-Platte und Spongiosainterposition in das ausgeräumte Bandscheibenfach. Aus OOT, Urban \& Schwarzenberg, München.

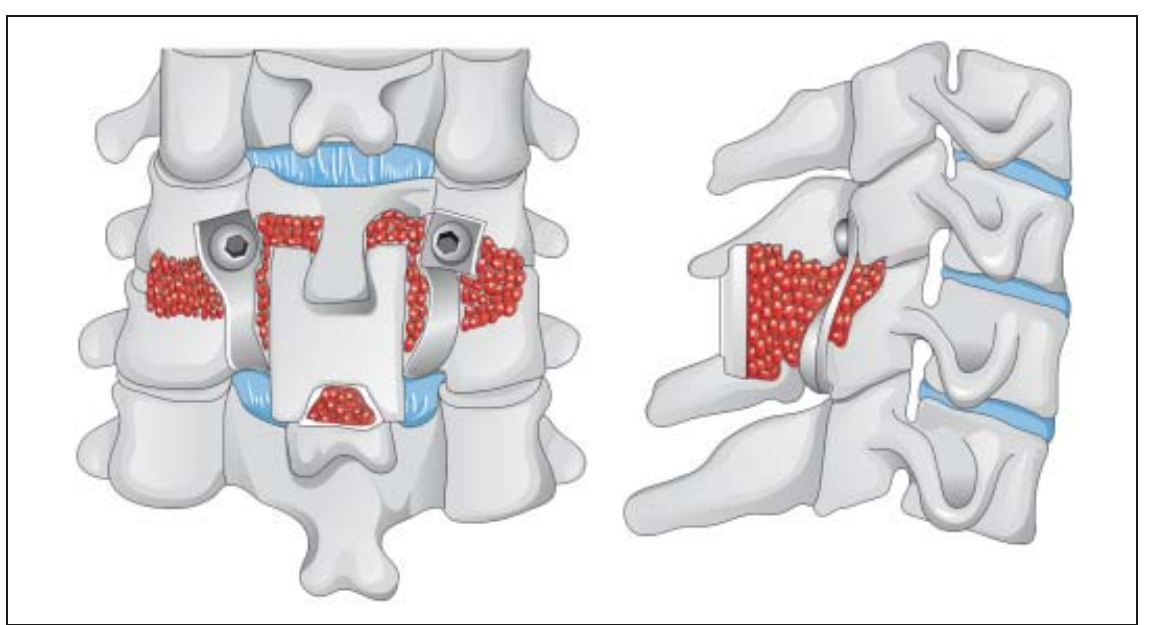

Abb.14 Dorsale Spondylodese mit Hakenplatte nach Mangel und Sponiosaanagerung. Aus OOT Urban \& Schwarzenberg, München.

Der ventrale Zugang ist schonender, da meist keine Muskeln durchtrennt werden müssen. Er führt zu einem geringeren Blutverlust als der dorsale Zugang. Er wird in Rückenlage durchgeführt, was die präoperative Reposition und intraoperative Stellungskorrektur erleichtert. Eine Umlagerung entfällt (instabile Verletzungen!). Die Rückenlage ist für ältere Verletzte und Verletzte mit Polytrauma schonender. Die Kompression des Myelons erfolgt meist von ventral, so dass die Dekompression von ventral sicherer möglich ist als von dorsal. Die Heilungsrate der ventralen Spondylodese ist höher als die der dorsalen.

Der dorsale Zugang erleichtert die Reposition von verhakten Luxationen der Gelenkfortsätze, die von ventral manchmal reicht werden kann und sich nach der ventralen Stabilisierung noch eine Restinstabilität bei der obligaten intraoperativen Bildwandlerkontrolle demonstrieren lässt. Dies kommt vor allem bei ausgeprägt instabilen Verletzungen der Segmente $\mathrm{C} 6 / \mathrm{C} 7$ und $\mathrm{C} 7 / \mathrm{Th} 1$ vor. Auch bei chronisch versteiften Wirbelsäulen, wie z.B. bei Morbus Bechterew, ist eine alleinige Versteifung von ventral manchmal nicht ausreichend (Abb.15).

Jede Versteifung eines Bewegungssegmentes führt zu einer Mehrbelastung der angrenzenden Segmente mit dem Risiko der frühzeitigen Degeneration. Daher ist das Ausmaß der Belastung abhängig von der Anzahl der versteiften Segmente. Es sollte möglichst immer nur das verletzte Segment versteift werden (Abb.16).

Gründe für eine Versteifung von zwei Segmenten sind:

- Der betroffene Wirbelkörper ist soweit zerstört, dass die Schrauben zur Platzierung der Platte keinen ausreichenden Halt finden.

- Bei der Ausräumung eines, das Myelom komprimierenden großen Fragments der Hinterkante des Wirbelkörpers muss soviel Substanz des Wirbelkörpers entfernt werden, dass die Schrauben im verletzten Wirbelkörper keinen Halt mehr finden.

- Insbesondere bei Verletzungen des Bewegungssegmentes $\mathrm{C} 2 / \mathrm{C} 3$, das von ventral relativ schwierig zu erreichen ist, muss manchmal um genügend Überblick über den Spinalkanal zu erreichen, ebenfalls so viel Substanz entfernt werden, dass die Platte nur im gesunden darunterliegenden Wirbelkörper verankert werden kann.

nicht möglich ist. Die Kompression einer Nervenwurzel resultiert meist aus einem verlagerten frakturierten Gelenkfortsatz, der besser von dorsal reponiert werden kann. Die seltene Kompression des Rückenmarks durch dorsale Strukturen wird einfacher und sicherer von dorsal entlastet. Bei manchen anatomischen $\mathrm{Ge}$ gebenheiten ist ein ventraler Zugang nur sehr schwer oder gar nicht möglich, z. B. bei ausgeprägten Kyphosen bei Morbus Bechterew, oder bei liegendem Tracheostoma (Infektionsgefahr!). Bis auf die Fälle mit diesen seltenen Befunden stabilisieren wir die Halswirbelsäule in der Regel von ventral.

Ein kombiniertes ventro-dorsales Vorgehen wählen wir immer dann, wenn von ventral keine ausreichende Stabilität er-
In diesen Fällen muss der Beckenkammwegungssegmente überbrücken, damit eine dauerhafte knöcherne Überbrü-

\section{Schlussfolgerung}

Verletzungen der HWS führen häufiger zur Instabilität als Verletzungen der BWS und LWS und sie sind häufiger mit neurologischen Komplikationen vergesellschaftet. Jeder Patient mit einer Halswirbelsäulenverletzung muss eingehend neurologisch untersucht und einer sorgfältigen Röntgenuntersuchung unterzogen werden. Die gesamte HWS vom Hinterhaupt bis zum Bewegungssegment C7/ Th1 muss dargestellt werden. Je nach Sispan die Bandscheibenfächer beider Beckung resultiert. 


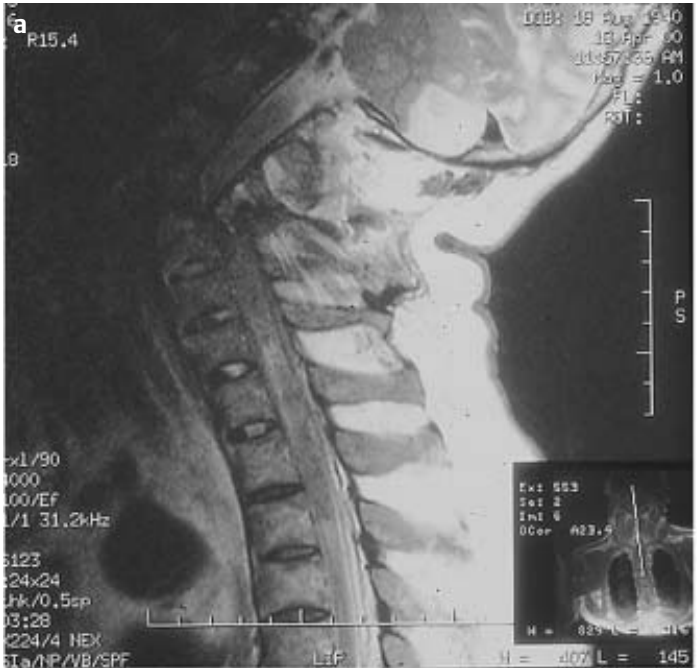

Abb.15 a MRT eines bewusstlosen Verletzten, der in angetrunkenem Zustand auf der Straße gestolpert war: Die HWS ist in Höhe C5 gebrochen und durch die Lagerung auf einem ebenen Tisch bei fixierter Ky-
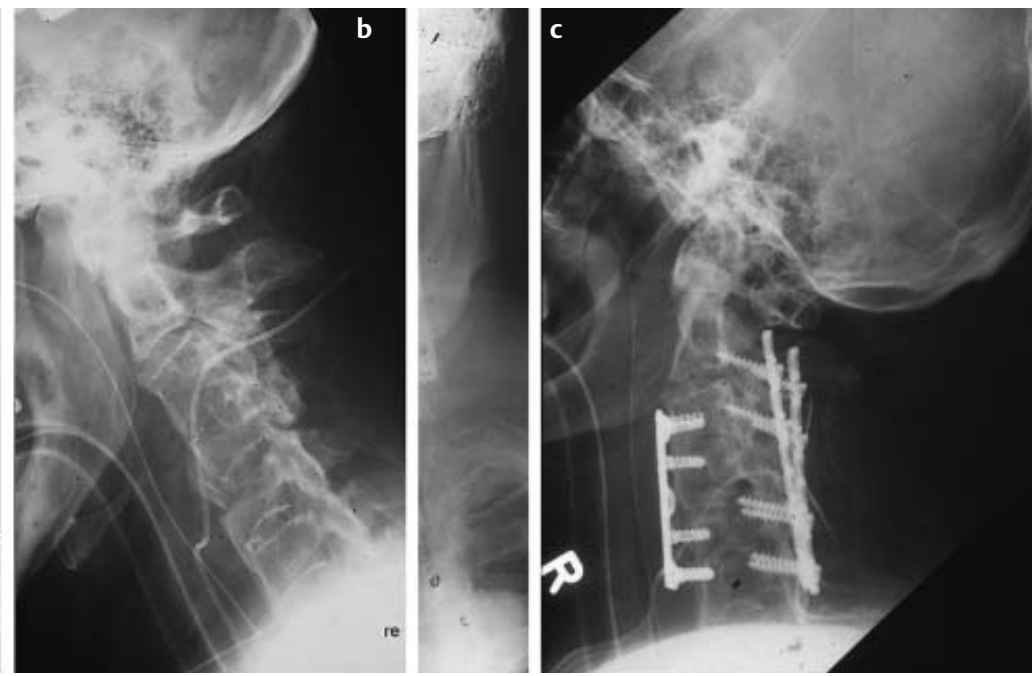

phose der Rest-HWS im Bruch um fast $70^{\circ}$ gekippt. b seitliches Röntgenbild nach Reposition durch Hochlagerung des Kopfes. c Um Stabilität zu erreichen, musste hier von dorsal und ventral stabilisiert werden.
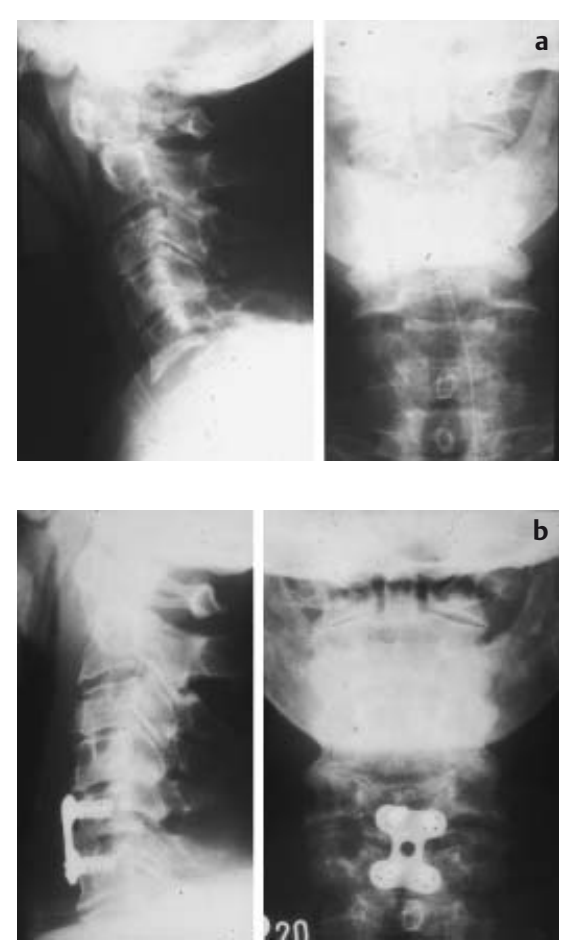

Abb.16 a Luxation C5/6; b monosegmentale Spondylodese $\mathrm{C} 5 / 6$. tuation sind weitere Schnittbilduntersuchungen nötig (CT, MRT). Die operative Therapie ist absolut indiziert bei Tetraplegie, bei mechanisch erklärbarer neurologischer Symptomatik und bei ausgeprägten Instabilitäten eines Bewegungssegmentes. Empfehlenswerte Operationsindikationen sind weniger ausgeprägte Instabilitäten, da die alternative konservative Behandlung mit 6-8 Wochen Halofixateur wenig komfortabel ist und nicht sicher zur stabilen Ausheilung führt.

Kompressionsfrakturen ohne Instabilität werden in der Zervikalstütze für wenige Wochen behandelt.

\section{Literatur}

${ }^{1}$ Benson DR, Keenen R. Evaluation and treatment of trauma to the vertebral column. Instructional Course Lecture 1990; 39: 577 589

2 Blauth M, Tscherne H. Untere HWS, In: $\mathrm{H}$. Tscherne, M. Blauth (Hrsg): Unfallchirurgie Wirbelsäule. Springer, Berlin 1998; 153 - 197
3 Crowell RR, Edward WT, WhitelII AA. Mechanism of injury in the cervicle spine: Experimental evidence and biomechanical modeling. In: The cervical spine research society editorial commitee: The cervical spine. Lippincott, Philadelphia 1989; 70-90

4 Magerl F, Aebi M, Gertzbein SD, Harms J, Nazarian S. A comprehensive classification of thoracic and lumbar injuries. Eur Spine J 1994; 3: 184-201

5 O'Dowd J. Spine. In Rüedi TP, Murphy WM: AO Principles of fracture management. Thieme Stuttgart 2000; 601-617

${ }^{6}$ White AA, Panjabi MM. The problem of clinical instability in the humane spine: A systematic approach. In: White AA, Panjabi MM (eds): Clinical biomechanics of the spine. Lippincott, Philadelphia 1990; 277-378

Prof. Dr. med. Ulrich Holz

Ärztlicher Direktor

Dr. med. Michael Oberst

Assistenzarzt

Dr. med. Bernd Wittner

Oberarzt

Katharinenhospital

Klinik für Unfall- und Wieder-

herstellungschirurgie

Kriegsbergstraße 60

D-70174 Stuttgart 